\title{
Synthesis and Ex Situ Doping of ZnTe and ZnSe Nanostructures with Extreme Aspect Ratios
}

\author{
Joanne W. L. Yim ${ }^{1,2}$, Deirdre Chen ${ }^{1}$, Gregory F. Brown ${ }^{1,2}$, and Junqiao $\mathrm{Wu}^{1,2}(\square)$ \\ ${ }^{1}$ Department of Materials Science and Engineering, University of California, Berkeley, CA 94720, USA \\ ${ }^{2}$ Materials Sciences Division, Lawrence Berkeley National Laboratory, Berkeley, CA 94720, USA \\ Received: 8 September 2009 / Revised: 8 October 2009 / Accepted: 12 October 2009 \\ CTsinghua University Press and Springer-Verlag 2009. This article is published with open access at Springerlink.com
}

\begin{abstract}
We report synthesis windows for growth of millimeter-long ZnTe nanoribbons and ZnSe nanowires using vapor transport. By tuning the local conditions at the growth substrate, high aspect ratio nanostructures can be synthesized. A Cu-ion immersion doping method was applied, producing strongly p-type conduction in ZnTe and ionic conduction in $\mathrm{ZnSe}$. These extreme aspect ratio wide-bandgap semiconductors have great potential for high density nanostructured optoelectronic circuits.
\end{abstract}

\section{KEYWORDS}

Aspect ratio, doping, nanowires, zinc selenide, zinc telluride

\section{Introduction}

Nanostructures, in particular "one-dimensional" structures such as nanowires and nanoribbons, present a useful materials platform for exploring quantum effects and fabricating novel devices at high densities. Free-carrier doping is needed for most of these applications. Typically, doping in nanowires is achieved by in situ incorporation of dopants during growth of the nanowires [1, 2]. For a wide range of nanowire device applications such as inversionmode transistors, avalanche photodiodes, light emitting diodes, and tunneling field-effect transistors, axially modulated doping is needed to accomplish or optimize their functionalities. Although axial modulation of doping profiles can be achieved in situ [3], accurate alignment and positioning of the appropriately doped regions of the nanowires to build functional devices are complex and challenging. Ex situ doping can avoid these complications by introducing specific dopants into lithographicallydefined regions after the nanowires are grown [4-6] or even after the devices are assembled [7].

ZnSe and ZnTe, with direct bandgaps at 2.65 and $2.25 \mathrm{eV}$, respectively, are ideally situated on the optical spectrum for green-blue optical devices as well as other optoelectronic applications $[8,9]$. ZnSe nanowires can also be used as nanoscale optical cavities [10]. Undoped nanowires of $\mathrm{ZnSe}$ and $\mathrm{ZnTe}$ with strong band-edge and defect luminescence have been grown by vapor transport and other methods [10-14]. Intentional doping of nanowires

Address correspondence towuj@berkeley.edu 
of these compounds is less common. For compound semiconductors, the overall stoichiometry must be regulated in addition to incorporating and activating dopants, making in situ doping a nontrivial task. Doping of ZnTe and ZnSe bulk and thin film materials, in particular making p-type ZnSe and n-type ZnTe, has historically been difficult due to compensation from native defects [15]. But some success has been achieved in thin films via molecular beam epitaxy [16] and in nanowires via chemical vapor deposition $[17,18]$. These nanowires are typically limited in length to tens of micrometers. For integration of individual devices into functional circuits, nanostructures with greater lengths and higher aspect ratios are much desired. In this work, we report a method of synthesizing and ex situ doping of ZnTe nanoribbons and ZnSe nanowires with extremely large aspect ratios. The lengths of ZnTe nanoribbons are up to hundreds of micrometers and of ZnSe nanowires are up to a few millimeters. Our ex situ doping using $\mathrm{Cu}$ ion immersion [6] results in successful p-type doping of $\mathrm{ZnTe}$ and ionic conduction in ZnSe. These ultra-long, single-crystal, ex situ doped group II-VI nanostructures could enable a wide range of studies of integrated electrical, optoelectronic, or photonic devices.

\section{Experimental}

\subsection{Synthesis}

$\mathrm{ZnTe}$ and $\mathrm{ZnSe}$ nanostructures were synthesized by gold-catalyzed vapor transport from ZnTe and ZnSe (Aldrich 99.99\%) powder sources, respectively. Oxidized silicon wafers were functionalized, and then covered with $20 \mathrm{~nm}$ Au colloids (Aldrich). The source powders were placed at the center of a $1^{\prime \prime}$ tube furnace, with the growth substrates downstream. After repeated flushing with Ar to remove residual oxygen, the nanoribbons and nanowires were grown for $1 \mathrm{~h}$ under flowing Ar. Optimal growth source temperature $(T)$, pressure $(P)$, time $(t)$, and carrier gas flow rate $\left(k_{\text {flow }}\right)$ for $\mathrm{ZnTe}$ nanoribbons were $T=800{ }^{\circ} \mathrm{C}, P=10$ torr, $t=1 \mathrm{~h}$, and $k_{\text {flow }}=50$ standard cubic centimeters per minute $(\mathrm{sccm})$. For ultra-long ZnSe nanowires, $\mathrm{T}=1050{ }^{\circ} \mathrm{C}, P=10$ torr, $t=1 \mathrm{~h}$, and $k_{\text {flow }}=100 \mathrm{sccm}$.

\subsection{Structural characterization}

The morphology and structure of synthesized nanostructures were studied by a Zeiss Gemini Ultra-55 Analytical scanning electron microscope (SEM) equipped with an energy dispersive X-ray spectroscopy (EDX) system, X-ray diffraction (Siemens D5000), and transmission electron microscopy (TEM, JEOL 2100F). For TEM studies, the nanowires were dispersed in isopropanol and deposited on a lacey carbon film supported on a $\mathrm{Cu}$ grid.

\subsection{Doping}

For ex situ doping, the nanoribbons and nanowires were transferred to $\mathrm{SiO}_{2}$ substrates. The substrates were immersed in an aqueous $\mathrm{Cu}\left(\mathrm{NO}_{3}\right)_{2}$ solution $[6,19,20]$. They were subsequently rinsed in deionized (DI) water and dried with compressed air. The samples were annealed at $400{ }^{\circ} \mathrm{C}$ under vacuum. The conditions used for $\mathrm{ZnTe}$ and $\mathrm{ZnSe}$ are given in Table 1. Two-probe and four-probe devices were fabricated on single nanoribbons or nanowires by photolithography and contact metals were deposited by electron-beam evaporation.

\section{Results and discussion}

\subsection{ZnTe nanoribbons}

Typical synthesized ZnTe nanoribbons ranged in size

Table 1 Cu-doping conditions and contact metal used

\begin{tabular}{cccccc}
\hline Material & $\begin{array}{c}\mathrm{Cu}\left(\mathrm{NO}_{3}\right)_{2} \text { in } 150 \mathrm{~mL} \\
\mathrm{Dl} \text {-water }(\mathrm{mg})\end{array}$ & $\begin{array}{c}\text { Solution } \\
\text { temperature }\left({ }^{\circ} \mathrm{C}\right)\end{array}$ & $\begin{array}{c}\text { Immersion duration } \\
(\mathrm{min})\end{array}$ & $\begin{array}{c}\text { Annealing temperature } \\
\text { /time }\left({ }^{\circ} \mathrm{C} / \mathrm{min}\right)\end{array}$ & $\begin{array}{c}\text { Contact metal } \\
(\mathrm{nm})\end{array}$ \\
\hline ZnTe & 50 & 25 & $0,5,10,15,20$ & $400 / 20$ & $\mathrm{Ni} / \mathrm{Au} \mathrm{15/240}$ \\
ZnSe & 800 & 60 & $0,5,10,30,60$ & $400 / 60$ & $\mathrm{Cu} / \mathrm{Au} \mathrm{20/200}$ \\
\hline
\end{tabular}


from 50-200 $\mu \mathrm{m}$ in length and 1-10 $\mu \mathrm{m}$ in width, and were approximately $100 \mathrm{~nm}$ thick. As seen in the SEM image (Fig. 1(a)), the nanoribbons were tapered, with the wider regions closer to the substrate. Au-rich balls can be found at the narrow ribbon tips (Fig. 1(c)), and their presence was confirmed by EDX spectroscopy. $X$-ray diffraction confirms that the nanoribbons are face-centered cubic (JCPDS-15-0746) (Fig. 1(b)). Highresolution TEM (HRTEM) shows that the major axis of the nanoribbon goes along [311] (Figs. 1(c) and $1(\mathrm{~d})$ ). The wide bounding facet of the nanoribbons is (110). Selected area electron diffraction (SAED) patterns (inset in Fig. 1(d)) along the length of the nanoribbons have identical orientation, indicating the single-crystallinity of the nanoribbons. These ZnTe nanoribbons have a strong and narrow bandedge emission peak at $2.25 \mathrm{eV}$ recorded by microphotoluminescence at room temperature (not shown), as well as a less prominent broad red luminescence that is commonly attributed to oxygen impurities.

Previous reports of tapered nanoribbons grown by vapor transport attribute the morphology to high source and substrate temperatures; the first increases the amount of molecules in the vapor and the second enhances the surface mobility of adsorbed molecules, allowing side growth and forming the ribbons [21].
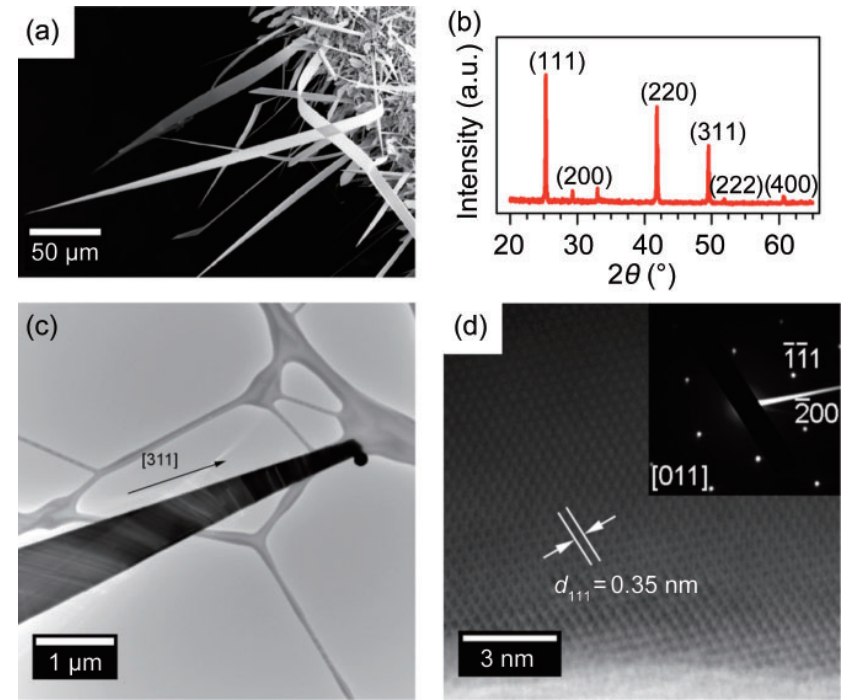

Figure 1 (a) SEM image of ZnTe nanoribbons as grown. (b) XRD pattern obtained from the as-grown chip, indexed to zinc blende ZnTe. (c) Low magnification TEM image of a ZnTe nanoribbon at a tapered region with Au catalyst tip still attached. (d) High-resolution TEM image with corresponding SAED (inset)
The length of the nanoribbons is controlled by the competition between side-wall and catalyst-enabled growth. For lower growth temperatures, molecules are more likely to be incorporated at nearby sites forming ribbons, rather than moving to the catalyst and extending the length.

These high aspect-ratio nanostructures provide a large surface area to volume ratio for fast ex situ doping and a convenient topology for defining electrical contacts, as was done by photolithography and subsequent metal deposition. The $\mathrm{Ni} / \mathrm{Au}$ contacts were ohmic, exhibiting linear current-voltage $(I-V)$ characteristics. With increasing $\mathrm{Cu}$ immersion time, the resistivity of the nanowires decreased from $10^{5}$ $\Omega \cdot \mathrm{cm}$ for the undoped case to nearly $1 \Omega \cdot \mathrm{cm}$ for the highly $\mathrm{Cu}$-doped samples (Fig. 2). In comparison to the un-annealed sample, vacuum annealing at $400{ }^{\circ} \mathrm{C}$ causes a slight increase in resistivity. Indeed, vacuum anneals have been shown to decrease the crystal quality of ZnTe [22]. The undoped nanoribbons have two orders of magnitude higher resistivity than reported values for comparable nanostructures [18], but the properties of our $\mathrm{Cu}$-doped nanoribbons agree well with those of doped nanowires [6]. The trend of decreasing resistivity with immersion time is in line with values obtained from similarly treated

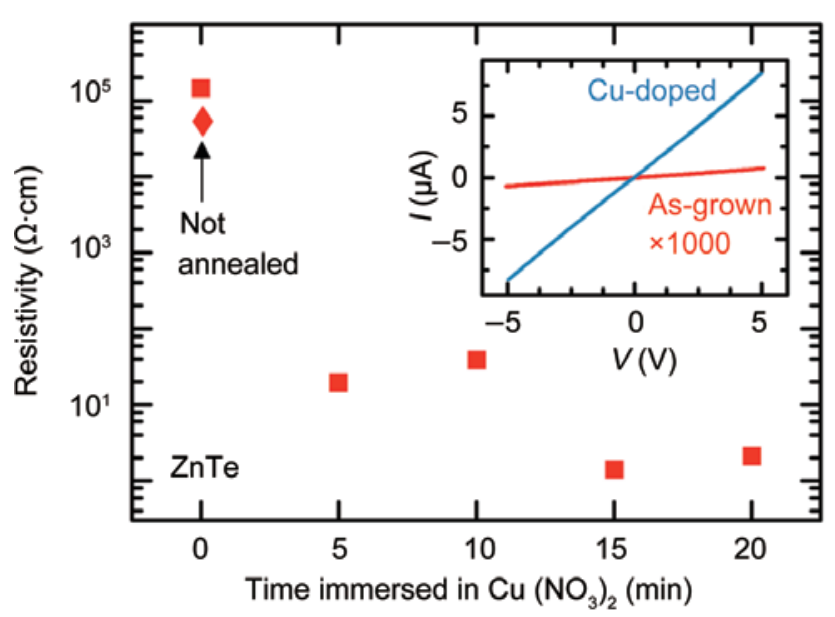

Figure 2 Resistivity of ZnTe nanoribbons versus immersion time in the $\mathrm{Cu}\left(\mathrm{NO}_{3}\right)_{2}$ solution. The samples were vacuum annealed at $400{ }^{\circ} \mathrm{C}$ for 20 min, except for a control case which was neither immersed nor annealed. (Inset) Linear I-V curves from an as-grown ZnTe nanoribbon and another which had been immersed for 20 min. The as-grown signal has been magnified by 1000 . The nanoribbon segment measured is approximately $5 \mu \mathrm{m}$ in width, $100 \mathrm{~nm}$ in thickness, and $20 \mu \mathrm{m}$ in length 
thin films $[19,20]$.

It is well established that $\mathrm{Cu}$ sitting substitutionally on Zn sites in ZnTe creates an acceptor level $149 \mathrm{meV}$ above the valence band maximum [23]. As expected, both doped and undoped nanoribbons exhibit p-type behavior, with slightly enhanced conduction on applied negative gate voltage (not shown). It has been proposed that the copper ions are incorporated through an ion exchange process with $\mathrm{Zn}$ at the surface, leading to the development of a $\mathrm{Cu}$ surface layer which eventually blocks the incorporation of additional $\mathrm{Cu}$ [19]. Based on optical transmission studies, vacuum annealing at $400{ }^{\circ} \mathrm{C}$ is sufficient to diffuse $\mathrm{Cu}$ throughout a $500 \mathrm{~nm}$ film [19]. In addition to occupying $\mathrm{Zn}$ sites, Cu dopants are reported to accumulate at grain boundaries and Te-inclusions [24]. However, our nanoribbons should not have such doping inhomogeneity due to their nanoscale dimensions and single-crystal nature.

\subsection{ZnSe nanowires}

Growth substrates of ZnSe nanowires emerged covered with a bright yellow mat of nanowires, even more brilliantly colored than the starting ZnSe source powder (Fig. 3(d)).Radii vary widely from $30 \mathrm{~nm}$ to several microns, and can have lengths up to a few millimeters. These extreme lengths lead to spectacular fields of nanowires when imaged by SEM and are visible even by the naked eye (Figs. 3(a) and 3(d)). The longer wires tend to be faceted and wider. Aspect ratios of length versus diameter of up to 10000 have been found. As a comparison, typical lengths of $\mathrm{ZnSe}$ nanowires grown by vapor transport have been previously reported to be a few to tens of microns [10, 12-14], with similar diameters to ours. While ZnSe nanowires grown by molecular beam epitaxy (MBE) are generally smaller in diameter (20-50 $\mathrm{nm}$ ), their aspect ratios are limited by lengths of only 1-2 $\mu \mathrm{m}$ [25]. In addition, the nanowires are constant in diameter along their length, as demonstrated by Fig. 3(e). XRD spectra of as-grown ZnSe nanowires show that the predominant polytype is zinc blende (JCPDS 37-1463), although there may be a small percentage of wurtzite (JCPDS 80-0008) (Fig. 3(b)). Au-tips are also visible at the top of some nanowires, as can be expected from the Au-catalyzed vapor-
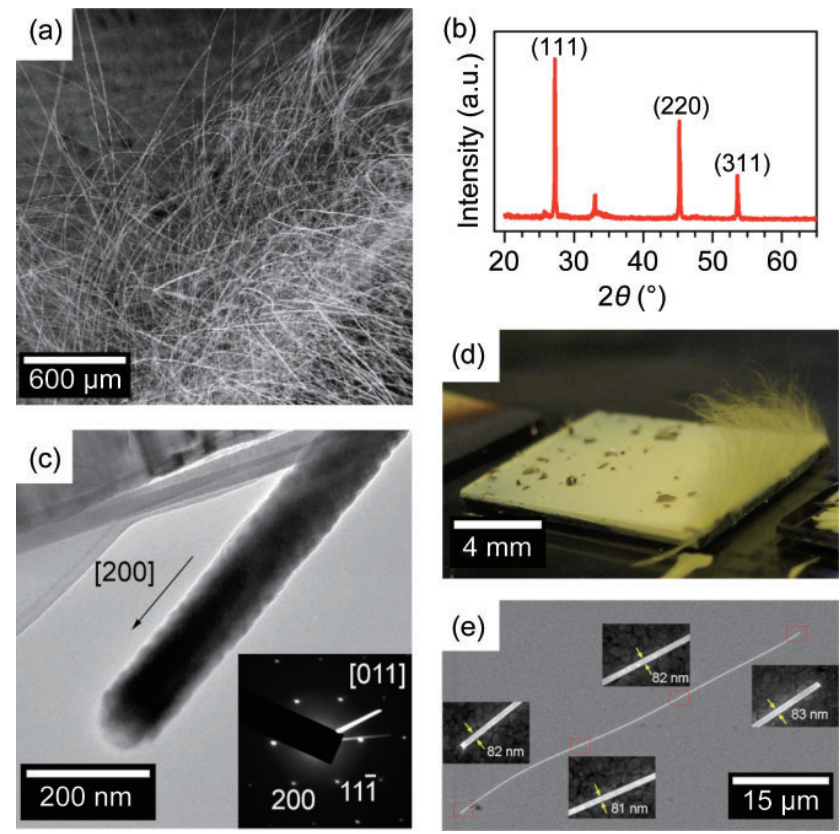

Figure 3 (a)SEM image of ZnSe nanowires as grown. (b) XRD pattern obtained from nanowires on the as-grown substrate, indexed to zinc blende ZnSe. (c) TEM image of a ZnSe nanowire with associated SAED (inset). (d) Photograph of growth substrate, where the $\mathrm{mm}$-long nanowires were grown on the upstream edge. (e) SEM image of a highly uniform ZnSe nanowire transferred onto an Au/Si substrate. The wire was broken during transfer, and is therefore shorter than as-grown structures. (Insets) SEM images of the endpoints and two middle segments

liquid-solid (VLS) growth.

Ultra-long nanowires of $\mathrm{Si}$ have been grown via VLS using $\mathrm{Si}_{2} \mathrm{H}_{6}$ as opposed to the more common $\mathrm{SiH}_{4}$ to overcome the rate-limiting step of precursor decomposition [26]. However, growth of ultra-long group II-VI nanowires has not been reported. In our case, increasing growth time at lower growth temperatures did not produce such ultra-long nanowires. We therefore attribute the growth of ultra-long ZnSe nanowires to the elevated source temperature $\left(1050{ }^{\circ} \mathrm{C}\right)$ compared with other reports $\left(950{ }^{\circ} \mathrm{C}\right)$, which provides sufficient vapor supply, and the elevated substrate temperature $\left(780^{\circ} \mathrm{C}\right)$ compared with other reports $\left(650-725^{\circ} \mathrm{C}\right)$, which increases surface mobility of adsorbed adatoms on nanowire side-walls, allowing them to rapidly diffuse to the Au-catalyst. Variations in the growth parameters, including decreasing growth temperature or adding a Zn source, also produce ZnSe nanowires; however, they do not reach the extreme lengths produced 
under the optimal conditions. Room-temperature micro-photoluminescence shows strong broad luminescence near $2.0 \mathrm{eV}$ and very weak band-edge luminescence near $2.65 \mathrm{eV}$. In previous reports of ZnSe nanowires, it was found that higher growth temperatures resulted in a higher prevalence of structural defects and thus more intense defect luminescence at room temperature [27]. This may be the reason for the high ratio of defect-to-band-edge luminescence in our nanowires.

The electrical response of $\mathrm{Cu}$-doped $\mathrm{ZnSe}$ nanowires developed non-linearity with increasing $\mathrm{Cu}$-ion immersion. Previous reports of undoped, vapor-transport grown ZnSe nanowires show resistivity ranging from 1 to $105 \Omega \cdot \mathrm{cm}[17,28]$, which reflects the importance of growth conditions to the electrical quality. Our undoped ZnSe nanowires, both as-grown and annealed, show linear $I-V$ characteristics with resistivities in the range $0.1-$ $1 \Omega \cdot \mathrm{cm}$. After $\mathrm{Cu}$-immersion, we saw no obvious improvement in conductivity in contrast to the $\mathrm{ZnTe}$ case; instead, a hysteretic resistive switching effect appeared, as shown in the inset of Fig. 4. In the switching behavior observed in oxide memristors [29], the device starts with a high-resistance state, and then sweeps back through a low resistance state. In contrast, in our Cu-doped $\mathrm{ZnSe}$ nanowire devices the hysteresis loop starts at a low-resistance state, and abruptly changes over to a high-resistance state when swept to a positive threshold bias. The switch occurs between 0.3 and $0.5 \mathrm{~V}$, varying slightly from device to device. When returning to zero bias and sweeping to negative bias, the nanowire behaves nearly symmetrically, starting in a low resistance and transferring to a higher-resistance state. A full understanding of the mechanism of such a resistance switching in $\mathrm{Cu}$-doped $\mathrm{ZnSe}$ needs further investigation. Previous work in $\mathrm{Cu}$ doped ZnSe highlights the intricacies involved in making $\mathrm{Cu}$ dopants active in $\mathrm{ZnSe}$. Cu-immersion of polycrystalline $\mathrm{ZnSe}$ thin films, similar in method to this work, incorporated up to $1 \mathrm{wt} \% \mathrm{Cu}$, causing the resistivity to drop eight orders of magnitude [30]. However, for co-evaporated $20 \% \mathrm{Cu}$ films, ex situ annealing made the film non-conductive [31]. 1\% $\mathrm{Cu}$ doping of $\mathrm{ZnSe}$ from the melt also showed an

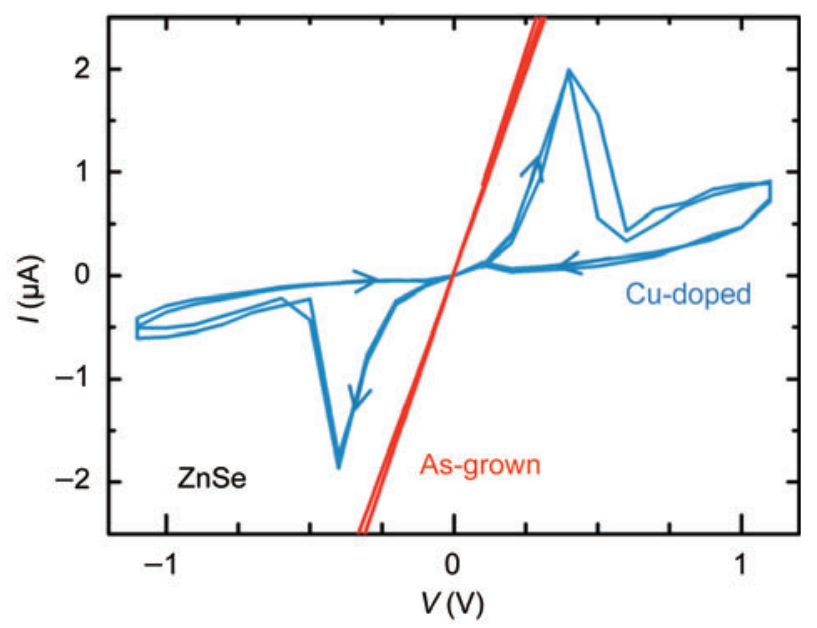

Figure 4 Typical I-V curves of as-grown and Cu-doped (30 min) ZnSe nanowires. The nanowire segment measured is approximately 100 $\mathrm{nm}$ in diameter and $4 \mu \mathrm{m}$ in length

increase in resistivity with increased $\mathrm{Cu}$ content [32]. It has been suggested that due to the multi-valence of $\mathrm{Cu}$ in $\mathrm{ZnSe}$, it is difficult to tune the Fermi level to make it highly conductive, since the Fermi level can be pinned between $\mathrm{Cu}$ deep levels at 0.72 and $0.35 \mathrm{eV}$ above the valence band maximum [33].

\section{Conclusions}

Crystalline ZnTe nanoribbons with lengths up to a millimeter and ZnSe nanowires with lengths exceeding several millimeters have been synthesized using the vapor transport method. We identified a narrow window of synthesis conditions that allow growth of such nanostructures with extreme aspect ratios. Utilizing a simple ion-immersion method, we ex situ doped these nanostructures with $\mathrm{Cu}$. Successful p-type doping was achieved in $\mathrm{ZnTe}$ nanoribbons where the resistivity was reduced by five orders of magnitude. $\mathrm{Cu}$ doping of $\mathrm{ZnSe}$ introduced a resistive switching effect, an electrical process involving ionic conduction possibly activated by the incorporation of multi-valent $\mathrm{Cu}$ dopants. Combined with their advanced optical and electrical properties, these ultra-long, single-crystal, ex situ doped group II-VI nanostructures could enable studies of nanostructure-based circuits integrating multiple electrical, optoelectronic, or photonic components. 


\section{Acknowledgements}

The authors thank Prof. A. Stacy of U. C. Berkeley for the use of the diffractometer. We are grateful for assistance from R. Chopkedar. The synthesis portion of this work was supported by the National Science Foundation (No. EEC-0832819), and the characterization portion by the Laboratory Directed Research and Development Program of Lawrence Berkeley National Laboratory under the Department of Energy (No. DE-AC02-05CH11231). J. Y. acknowledges support from the National Science Foundation Graduate Research Fellowship Program. Portions of this work were performed at the Molecular Foundry, LBNL, and the U. C. Berkeley Microfabrication Laboratory.

\section{References}

[1] Wang, Y. F.; Lew, K. K.; Ho, T. T.; Pan, L; Novak S. W.; Dickey, E. C.; Redwing, J. M.; Mayer, T. S. Use of phosphine as an n-type dopant source for vapor-liquidsolid growth of silicon nanowires. Nano Lett. 2005, 5, 2139-2143.

[2] Zhong, Z. H.; Fang, Y.; Lu, W.; Lieber, C. M. Coherent single charge transport in molecular-scale silicon nanowires. Nano Lett. 2003, 3, 343-346.

[3] Gudiksen, M. S.; Lauhon, L. J.; Wang, J.; Smith, D. C.; Lieber, C. M. Growth of nanowire superlattice structures for nanoscale photonics and electronics. Nature 2002, 415, 617-620.

[4] Colli, A.; Fasoli, A.; Ronning, C.; Pisana, S.; Piscanec, S.; Ferrari, A. C. Ion beam doping of silicon nanowires. Nano Lett. 2008, 8, 2188-2193.

[5] Ho, J. C.; Yerushalmi, R.; Jacobson, Z. A.; Fan, Z.; Alley, R. L.; Javey, A. Controlled nanoscale doping of semiconductors via molecular monolayers. Nat. Mater. 2008, 7, 62-67.

[6] Huo, H. B.; Dai, L.; Liu, C.; You, L. P.; Yang, W. Q.; Ma, R. M.; Ran, G. Z.; Qin, G. G. Electrical properties of Cu doped p-ZnTe nanowires. Nanotechnology 2006, 17, 5912-5915.

[7] Misra, N.; Grigoropoulos, C. P.; Stumbo, D. P.; Miller, J. N. Laser activation of dopants for nanowire devices on glass and plastic. Appl. Phys. Lett. 2008, 93, 121116.

[8] Gunshor, R. L.; Nurmikko, A. V. The first compact blue/ green diode lasers-wide-bandgap II-VI semiconductors come of age. Proc. IEEE 1994, 82, 1503-1513.

[9] Löffler, T.; Hahn, T.; Thomson, M.; Jacob, F.; Roskos, H. Large-area electro-optic ZnTe terahertz emitters. Opt. Express 2005, 13, 5353-5362.

[10] van Vugt, L. K.; Zhang, B.; Piccione, B.; Spector, A. A.; Agarwal, R. Size-dependent waveguide dispersion in nanowire optical cavities: Slowed light and dispersionless guiding. Nano Lett. 2009, 9, 1684-1688.

[11] Huo, H. B.; Dai, L.; Xia, D. Y.; Ran, G. Z.; You, L. P.; Zhang, B. R.; Qin, G. G. Synthesis and optical properties of ZnTe single-crystalline nanowires. J. Nanosci. Nanotechnol. 2006, 6, 1182-1184.

[12] Philipose, U.; Yang, S.; Xu, T.; Ruda, H. E. Origin of the red luminescence band in photoluminescence spectra of ZnSe nanowires. Appl. Phys. Lett. 2007, 90, 063103.

[13] Xiang, B.; Zhang, H. Z.; Li, G. H.; Yang, F. H.; Su, F. H.; Wang, R. M.; Xu, J.; Lu, G. W.; Sun, X. C.; Zhao, Q.; Yu, D. P. Green-light-emitting ZnSe nanowires fabricated via vapor phase growth. Appl. Phys. Lett. 2003, 82, 33303332.

[14] Ye, C.; Fang, X.; Wang, Y.; Yan, P.; Zhao, J.; Zhang, L. Structural characterization of long ZnSe nanowires. Appl. Phys. A: Mater. Sci. Process. 2004, 79, 113-115.

[15] Chadi, D. J. The problem of doping in II-VI semiconductors. Ann. Rev. Mater. Sci. 1994, 24, 45-62.

[16] Baron, T.; Saminadayar, K.; Magnea, N. Nitrogen doping of Te-based II-VI compounds during growth by molecular beam epitaxy. J. Appl. Phys. 1998, 83, 1354-1370.

[17] Song, H. S.; Zhang, W. J.; Yuan, G. D.; He, Z. B.; Zhang, W. F.; Tang, Y. B.; Luo, L. B.; Lee, C. S.; Bello, I.; Lee, S. T. p-type conduction in arsenic-doped ZnSe nanowires. Appl. Phys. Lett. 2009, 95, 033117.

[18] Zhang, J.; Chen, P. -C.; Shen, G.; He, J.; Kumbhar, A.; Zhou, C.; Fang, J. p-type field-effect transistors of singlecrystal zinc telluride nanobelts. Angew. Chem. Int. Edit. 2008, 47, 9469-9471.

[19] Aqili, A. K. S.; Maqsood, A.; Ali, Z. Properties of copperdoped ZnTe thin films by immersion in Cu solution. Appl. Surf. Sci. 2001, 180, 73-80.

[20] Maqsood, A.; Shafique, M. Properties of Cu-doped ZnTe thin films prepared by closed space sublimation (CSS) techniques. J. Mater. Sci. 2004, 39, 1101-1103.

[21] Fasoli, A.; Colli, A.; Hofmann, S.; Ducati, C.; Robertson, J.; Ferrari, A. C. Shape-selective synthesis of $I I-V I$ semiconductor nanowires. Phys. Status Solidi B 2006, 
243, 3301-3305.

[22] Kobayashi, M.; Terakado, H.; Sawada, R.; Arakawa, A.; Sato, K. Diffusion profiles of Se in bulk ZnTe. Physica $B$ 2002, 229, 265-268.

[23] Magnea, N.; Bensahel, D.; Pautrat, J. L.; Saminadayar, K.; Pfister, J. C. Electrical and opitical-indentification of the presistent acceptor as copper in ZnTe. Solid State Commun. 1979, 30, 259-263.

[24] Bensahel, D.; Magnea, N.; Dupuy, M. Behavior of copper in ZnTe SEM CL and PL. Solid State Commun. 1979, 30, 467-472.

[25] Aichele, T.; Tribu, A.; Bougerol, C.; Kheng, K.; Andre, R.; Tatarenko, S. Defect-free ZnSe nanowire and nanoneedle nanostructures. Appl. Phys. Lett. 2008, 93, 143106.

[26] Park, W. I.; Zheng, G.; Jiang, X.; Tian, B.; Lieber, C. M. Controlled synthesis of millimeter-long silicon nanowires with uniform electronic properties. Nano Lett. 2008, 8, 3004-3009.

[27] Wang, Y. Q.; Philipose, U.; Xu, T.; Ruda, H. E.; Kavanagh, K. L. Twinning modulation in ZnSe nanowires. Semicond. Sci. Technol. 2007, 22, 175-178.
[28] Salfi, J.; Philipose, U.; de Sousa, C. F.; Aouba, S.; Ruda, H. E. Electrical properties of Ohmic contacts to ZnSe nanowires and their application to nanowire-based photodetection. Appl. Phys. Lett. 2006, 89, 261112.

[29] Strukov, D. B.; Snider, G. S.; Stewart, D. R.; Williams, R. S. The missing memristor found. Nature 2008, 453, 80-83.

[30] Ali, Z.; Aqili, A. K. S.; Maqsood, A.; Akhtar, S. M. J. Properties of $\mathrm{Cu}$-doped low resistive ZnSe films deposited by two-sourced evaporation. Vacuum 2005, 80, 302309.

[31] Orita, M.; Narushima, T.; Yanagita, H. Transparent conductive Cu-doped ZnSe film deposited at room temperature using compound sources followed by laser annealing. Jpn. J. Appl. Phys. 2007, 46, L976-L978.

[32] Bolboshenko, V. Z.; Djouadi, D.; Kasiyan, V. A.; Nedeoglo, D. D. Transport phenomena and photoconductivity relaxation in copper-doped zinc selenide crystals. Phys. Status Solidi A 1992, 133, 121-136.

[33] Stringfellow, G. B.; Bube, R. H. Photoelectronic properties of ZnSe crystals. Phys. Rev. 1968, 171, 903-915. 